\title{
Oleanolic acid inhibits colorectal cancer angiogenesis in vivo and in vitro via suppression of STAT3 and Hedgehog pathways
}

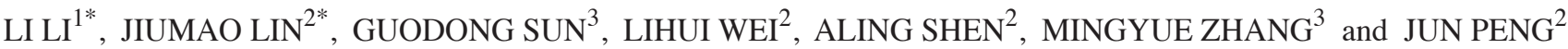 \\ ${ }^{1}$ Department of Disease Prevention and Healthcare, Fujian Provincial Hospital, Fuzhou, Fujian 350001; \\ ${ }^{2}$ Academy of Integrative Medicine, Fujian University of Traditional Chinese Medicine, Fuzhou, Fujian 350122; \\ ${ }^{3}$ Hangzhou Naval Sanatorium of Nanjing Military Area Command, Hangzhou, \\ Zhejiang 310002, P.R. China
}

Received May 27, 2015; Accepted April 13, 2016

DOI: $10.3892 / \mathrm{mmr} .2016 .5171$

\begin{abstract}
Angiogenesis is an essential process of cancer progression and is regulated by multiple intracellular signaling pathways, including signal transducer and activator of transcription 3 (STAT3) and sonic hedgehog (SHH). Thus, these pathways have become a promising target for anti-cancer therapeutic strategies. Oleanolic acid (OA) is an active compound present in various herbal medicines, which have been used historically for the clinical treatment of various types of human malignancies, including colorectal cancer (CRC). The present study used a CRC mouse xenograft model and human umbilical vein endothelial cells (HUVECs) to evaluate the effect of OA on tumor angiogenesis and on the activation of the STAT3 and $\mathrm{SHH}$ signaling pathways. It was determined that $\mathrm{OA}$ treatment significantly inhibited tumor growth and reduced intratumoral microvessel density (MVD) in CRC mice. In addition, $\mathrm{OA}$ treatment inhibited the proliferation, migration and tube formation in HUVECs, in a dose and time-dependent manner. Furthermore, OA markedly suppressed the activation of the STAT3 and SHH signaling pathways and inhibited the
\end{abstract}

Correspondence to: Dr Mingyue Zhang, Hangzhou Naval Sanatorium of Nanjing Military Area Command, 76 Yuhuangshan Road, Hangzhou, Zhejiang 310002, P.R. China

E-mail: zhangmy41501@163.com

Professor Jun Peng, Academy of Integrative Medicine, Fujian University of Traditional Chinese Medicine, 1 Qiuyang Road, Minhou Shangjie, Fuzhou, Fujian 350122, P.R. China

E-mail: pjunlab@hotmail.com

Abbreviations: OA, oleanolic acid; CRC, colorectal cancer; STAT3, signal transducer and activator of transcription 3; SHH, sonic hedgehog; MVD, microvessel density; VEGF-A, vascular endothelial growth factor A; bFGF, basic fibroblast growth factor; IHS, immunohistochemical staining

${ }^{*}$ Contributed equally

Key words: oleanolic acid, herbal medicine, signaling pathway, colorectal cancer, angiogenesis expression of the pro-angiogenic vascular endothelial growth factor A and basic fibroblast growth factor, two important target genes of the aforementioned signaling pathways. Therefore it is suggested that inhibition of tumor angiogenesis via the suppression of multiple signaling pathways may be one of the underlying mechanisms by which OA exerts its anti-cancer effect.

\section{Introduction}

Formation of new blood vessels via angiogenesis supports the continued growth of a solid tumor and promotes hematogenous metastasis, thus it is critical for cancer progression (1-5). The process of angiogenesis is mediated by multiple intracellular signaling pathways, including signal transducer and activator of transcription 3 (STAT3) and sonic hedgehog (SHH) transduction cascades (6-15). Aberrant activation of these pathways promotes tumor angiogenesis by inducing the expression of various critical angiogenic stimulators (16-20), including vascular endothelial growth factor A (VEGF-A) and basic fibroblast growth factor (bFGF). Thus, inhibition of tumor angiogenesis via suppression of the STAT3 and SHH signaling pathways may be a promising strategy for future cancer therapeutic strategies.

Natural products, including traditional Chinese medicines, have been used as anti-tumor treatments in China for thousands of years. They provide an alternative therapeutic strategy for a variety of diseases and result in relatively few adverse effects when compared with modern chemotherapeutic agents (21-23). Oleanolic acid (3ß-hydroxy-olea-12-en-28-oic acid, OA) is the principal active compound present in various traditional Chinese medicinal herbs (including Hedyotis diffusa and Patrinia scabiosaefolia) that have been used to clinically treat various types of human malignancies (24-27). Previous studies have reported that OA may suppress tumor growth via inhibition of proliferation and promotion of apoptosis of cancer cells (28-30). To further elucidate the underlying mechanism of anti-cancer activity, the present study used a colorectal cancer (CRC) mouse xenograft model and human umbilical vein endothelial cells (HUVECs) to evaluate the effect of OA on tumor angiogenesis and the activation of the STAT3 and SHH signaling pathways. 


\section{Materials and methods}

Materials and reagents. Oleanolic acid (OA) was purchased from Sigma-Aldrich (St. Louis, MO, USA). Matrigel was provided by BD Biosciences (San Jose, CA, USA). Dulbecco's modified Eagle's medium (DMEM), RPMI 1640, fetal bovine serum (FBS), penicillin-streptomycin, trypsin-EDTA, and TRIzol reagent were purchased from Thermo Fisher Scientific, Inc. (Waltham, MA, USA). PrimeScript RT Reagent kit was purchased from Takara Bio, Inc. (Otsu, Japan). Goldview Nucleic Acid Gel Stain (cat. no. G8142) was purchased from Beijing Solarbio Science \& Technology Co., Ltd. (Beijing, China). The In Vitro Angiogenesis assay kit was purchased from EMD Millipore (Billerica, MA, USA). BCA Protein assay kit was purchased from Tiangen Biotech Co., Ltd. (Beijing, China).

Cell culture. HT-29 human colon carcinoma cells were obtained from the Cell Bank of Chinese Academy of Sciences (Shanghai, China). Human umbilical vein endothelial cells (HUVECs) were purchased from Xiangya Cell Center, University of Zhongnan (Hunan, China). HT-29 cells and HUVECs were respectively cultured in DMEM or RPMI 1640 , at $37^{\circ} \mathrm{C}$ and $5 \% \mathrm{CO}_{2}$ in a humidified environment. DMEM and RPMI 1640 were supplemented with 10\% (v/v) FBS, 100 U/ml penicillin, and $100 \mu \mathrm{g} / \mathrm{ml}$ streptomycin.

In vivo nude mice xenograft study. A total of 20 six-week-old male BALB/c athymic (nude) mice (weight, 20-22 g) were obtained from Shanghai SLAC Laboratory Animal Co., Ltd. (Shanghai, China) and housed in a specific pathogen-free controlled environment (temperature, $22^{\circ} \mathrm{C} ; 12 \mathrm{~h}$ light/dark cycle) with ad libitum access to food and water. The HT-29 xenograft mouse model was generated as previously described (25). Following xenograft implantation, mice were placed in two treatment groups $(n=10)$ and treated with $12.5 \mathrm{mg} / \mathrm{kg}$ OA [dissolved in phosphate-buffered saline (PBS)] or saline by daily intraperitoneal injections, 6 days a week for 16 days. Body weight and tumor size were assessed. Tumor size was determined by measuring the major (L) and minor (W) tumor diameter with calipers. The tumor volume (T) was calculated according to the following formula: $\mathrm{T}=\pi / 6 \mathrm{x} \mathrm{Lx} \mathrm{W}^{2}$. At the end of the experiment, animals were sacrificed using pelltobarbitalum natricum overdose (Sigma-Aldrich), and tumors were excised. A portion of each tumor $\left(0.125 \mathrm{~cm}^{3}\right)$ was fixed in $10 \%$ buffered formalin (China National Medicines Corporation, Ltd., Beijing, China) and the remaining tissue was snap-frozen in liquid nitrogen and stored at $-80^{\circ} \mathrm{C}$. All animal treatments were performed in accordance with international ethical guidelines and the National Institutes of Health Guide for the Care and Use of Laboratory Animals (31). The experiments were approved by the Institutional Animal Care and Use Committee of Fujian University of Traditional Chinese Medicine (Fuzhou, China).

Immunohistochemical analysis. Slides of paraffin-embedded tumor tissue ( $\mathrm{n}=8 ; 4 \mu \mathrm{m}$; RM2235; Leica Microsystems GmbH, Wetzlar, Germany) were randomly selected from OA-treated or control groups, and analyzed by immunohistochemistry as previously described (25). Samples were blocked using normal goat serum in PBS with 0.1\% Tween 20 (Shanghai Sangon Biological Engineering Technology Co., Ltd., Shanghai, China), then incubated with rabbit polyclonal antibodies against cluster of differentiation (CD)31 (cat. no. 3528S; Cell Signaling Technology, Danvers, MA, USA), VEGF-A (cat. no. sc-7269; Santa Cruz Biotechnology, Inc.), bFGF (cat. no. 8910LC; Cell Signaling Technology), SHH (cat. no. sc-1194; Santa Cruz Biotechnology, Inc.) and GLI-Kruppel family member GLI1 (Gli-1; cat. no. sc-6152; Santa Cruz Biotechnology, Inc.) (all 1:200) were used to detect the relevant proteins by incubation overnight at $4^{\circ} \mathrm{C}$. Five fields (magnification, x400; DM4000; Leica Microsystems $\mathrm{GmbH}$ ) were randomly selected from each slide and the proportion of positive cells in each field was determined using a true color multi-functional cell image analysis management system (Image-Pro Plus version 6.0; Media Cybernetics, Rockville, MD, USA). PBS served as a negative control by replacing the primary antibody.

Cell viability evaluation by MTT assay. HUVECs were seeded into 96-well plates at a density of $1 \times 10^{4}$ cells/well in $0.1 \mathrm{ml} \mathrm{RPMI} 1640$ and were incubated for $24 \mathrm{~h}$ at $37^{\circ} \mathrm{C}$. In order to test dose-dependent effects of OA, the cells were treated with $0,25,50$ and $100 \mu \mathrm{M}$ OA [dissolved in $0.1 \%$ dimethyl sulfoxide (DMSO); Sigma-Aldrich] for $24 \mathrm{~h}$. In order to analyze time-dependent effects, with $40 \mu \mathrm{M}$ of OA for $0,1,3,6,12,8$ and $24 \mathrm{~h}$. At the end of the treatment, $10 \mu \mathrm{l}$ of $5 \mathrm{mg} / \mathrm{ml} \mathrm{MTT} \mathrm{(Sigma-Aldrich)} \mathrm{was} \mathrm{added} \mathrm{to} \mathrm{each}$ well, the samples were then incubated for an additional $4 \mathrm{~h}$. The formazan precipitate was dissolved in $100 \mu \mathrm{l}$ DMSO. Absorbance was measured at $570 \mathrm{~nm}$ using an single channel filter-based absorbance reader (ELx800; BioTek Instruments, Inc., Winooski, VT, USA).

Migration assay of HUVECs. Investigation into the migration of HUVECs was performed using the wound healing method. HUVECs were seeded into 12 -well plates at a density of $2 \times 10^{5}$ cells/well in $1 \mathrm{ml}$ medium. After $24 \mathrm{~h}$ of incubation, cells were scraped away vertically in each well using a P100 pipette tip. Three randomly selected views along the scraped line were photographed in each well using phase-contrast inverted microscopy at a magnification of x100 (FMIL/DFC295; Leica Microsystems GmbH). Following treatments with $\mathrm{OA}$ at various concentrations for $24 \mathrm{~h}$, a second set of images was captured using the same method. The reduction in the size of the scraped region is indicative of cell migration.

Tube formation assay of HUVECs. Tube formation in HUVECs was investigated using the In Vitro Angiogenesis assay kit (ECMatrix assay kit; EMD Millipore) following the manufacturer's protocol. Confluent HUVECs were harvested and diluted ( $1 \times 10^{4}$ cells) in $50 \mathrm{ml}$ RPMI 1640 containing various concentrations of OA. The harvested cells were seeded into 96-well plates with ECMatrix gel (1:1 v/v) and incubated for $9 \mathrm{~h}$ at $37^{\circ} \mathrm{C}$. The cells were photographed using phase-contrast inverted microscopy at a magnification of x100 (DMIL/DFC295; Leica Microsystems GmbH). The level of HUVEC tube formation was quantified by calculating the length of the tubes in three randomly selected fields from each well. 
Reverse transcription-polymerase chain reaction (RT-PCR). Total RNA was isolated from tumor tissues (3 tumors were randomly selected from OA-treated or control groups) or HT-29 cells with TRIzol reagent. Oligo(dT)-primed RNA (1 $\mu \mathrm{g}$, isolated from tumor tissues or cells) was reverse transcribed using the PrimeScript RT Reagent kit according to the manufacturer's protocol. Briefly, $1 \mu 1 \mathrm{gDNA}$ Eraser was used to remove the genomic DNA following incubation with total RNA for $2 \mathrm{~min}$ at $42^{\circ} \mathrm{C}$. Then, the PrimeScript RT Enzyme Mix and RT Primer Mix were added to perform the reverse transcription, by incubation for $15 \mathrm{~min}$ at $37^{\circ} \mathrm{C}$. The obtained cDNA was used to determine the mRNA expression levels of VEGF-A, bFGF, SHH and Gli-1 by PCR with Taq polymerase (Fermentas; Thermo Fisher Scientific, Inc.). The primers used for amplification of VEGF-A, bFGF, SHH, Gli-1, and GAPDH transcripts were as follows: VEGF-A forward, 5'-CATCCTGGCCTCGCTGTC-3' and reverse, 5'-CTCGCTCCAACCGACTGC-3'; bFGF forward, 5'-CGGCTGTACTGCAAAAACGG-3' and reverse, 5'-GATGTGAGGGTCGCTCTTCTCC-3'; SHH forward, 5'-CGGAGCGAGGAAGGGAAAG-3' and reverse, 5'-TTGGGGATAAACTGCTTGTAGGC-3'; Gli-1 forward, 5'-TCTGCCCCCATTGCCCACTTG-3' and reverse, 5'-TACATAGCCCCCAGCCCATACCTC-3'; and GAPDH forward, 5'-GTCATCCATGACAACTTTGG-3' and reverse, 5'-GAGCTTGACAAAGTGGTCGT-3'. The thermal cycling conditions were as follows: Denaturation at $95^{\circ} \mathrm{C}$ for $30 \mathrm{sec}$, annealing at the appropriate temperature (VEGF-A and GAPDH at $58^{\circ} \mathrm{C}$, bFGF at $56^{\circ} \mathrm{C}$, and $\mathrm{SHH}$ and Gli- 1 at $54^{\circ} \mathrm{C}$ ) for $30 \mathrm{sec}$, and extension at $60^{\circ} \mathrm{C}$ for $30 \mathrm{sec}$ for 30 cycles. GAPDH was used as an internal control. A negative control with no DNA and an RT control with no reverse transcription were used as the experimental controls. The PCR was repeated in 3 independent times. A BIO-RAD S1000 Thermal Cycler (Bio-Rad Laboratories, Inc., Hercules, CA, USA) was used to perform the experiment. Samples were analyzed by $1.5 \%$ agarose gel electrophoresis. The DNA bands were examined using a gel documentation system (Gel Doc 2000; Bio-Rad Laboratories, Inc.).

Western blotting. Protein from tumor tissues (3 tumors were randomly selected from OA-treated or control groups) and HT-29 cells was extracted using radioimmunoprecipitation assay protein extraction kit (Tiangen Biotech Co., Ltd.) containing protease and phosphatase inhibitor cocktails. For the interleukin (IL)-6 stimulation experiment, HT-29 cells were grown in complete DMEM (10\% FBS) until 70\% confluency, then cultured in FBS-free medium overnight. In complete DMEM, cells were pre-treated with various concentrations of OA for $1 \mathrm{~h}$ followed by stimulation with $10 \mathrm{ng} / \mathrm{ml}$ of IL-6 for $15 \mathrm{~min}$. The concentration of proteins was determined using the BCA Protein Assay Reagent kit. Proteins $(50 \mu \mathrm{g})$ were separated by $10 \%$ SDS-PAGE and transferred onto polyvinylidene fluoride membranes. The membranes were blocked for $1 \mathrm{~h}$ with $5 \%$ nonfat milk and incubated with the relevant primary antibody against STAT3 (cat. no. 9132), CD31 (cat. no. 3528S), phosphorylated STAT3 (cat. no. 9131), $\beta$-actin (cat. no. 4967) (all purchased from Cell Signaling Technology), SHH (sc-1194), VEGF-A (cat. no. sc-7269) or Gli-1 (cat. no. sc-6152) (all purchased

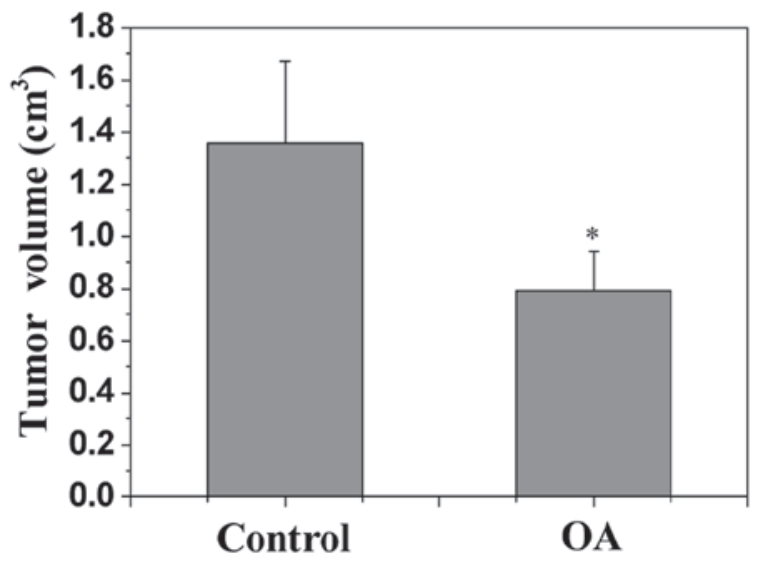

Figure 1. OA inhibited tumor growth in colorectal cancer xenograft mice. Tumor volume was determined following OA treatment. Data are presented as the mean \pm standard deviation from 10 individual mice from each group. ${ }^{*} \mathrm{P}<0.05$ vs. control group. OA, oleanolic acid.

from Santa Cruz Biotechnology, Inc.) overnight at $4^{\circ} \mathrm{C}$ (all in 1:1,000 dilutions). $\beta$-actin served as an internal control. The horseradish peroxidase-conjugated goat anti-rabbit secondary antibody (1:2,000 dilution; cat. no. E030120-01; EarthOx Life Sciences, Millbrae, CA, USA) were added for $1 \mathrm{~h}$ at room temperature. The levels of protein expression were detected with Enhanced Chemiluminescence (Beyotime Institute of Biotechnology, Haimen, China) detection. The chemiluminescence signals were visualized using the SuperSignal West Pico Chemiluminescent Substrate (Thermo Fisher Scientific, Inc.). Image Lab Software, version 3.0, was used for densitometric analysis of the western blotting (Bio-Rad Laboratories, Inc.).

Statistical analysis. Data were presented as mean \pm standard deviation for the indicated number of independently performed experiments. The data were analyzed using SPSS (version 17.0; SPSS, Inc., Chicago, IL, USA). Statistical analysis was performed on the data using Student's t-test and analysis of variance and $\mathrm{P}<0.05$ was considered to indicate a statistically significant difference.

\section{Results}

OA inhibits CRC angiogenesis. Tumor growth in CRC xenograft mice was evaluated by determining the volume of the tumor in the mice. As indicated by Fig. 1, OA treatment resulted in a significant reduction in tumor volume in CRC mice compared with the control $(\mathrm{P}<0.05)$, demonstrating the in vivo anti-tumor effect of OA. The in vivo tumor angiogenesis [intratumoral microvessel density (MVD)] was assessed by the expression of CD31, an endothelial cell-specific marker, using immunohistochemical staining. As indicated by Fig. 2A, OA significantly reduced the percentage of CD31-positive cells in CRC xenograft tumor tissues compared with the control $(\mathrm{P}<0.01)$, indicating its in vivo anti-angiogenic activity. The effect of OA on in vitro angiogenesis was also evaluated. As indicated by Fig. 2B-D, OA treatment significantly decreased the proliferation (viability) compared with the control $(\mathrm{P}<0.01)$, and decreased migration and capillary tube formation of HUVECs in dose- and/or time-dependent manner. 
A

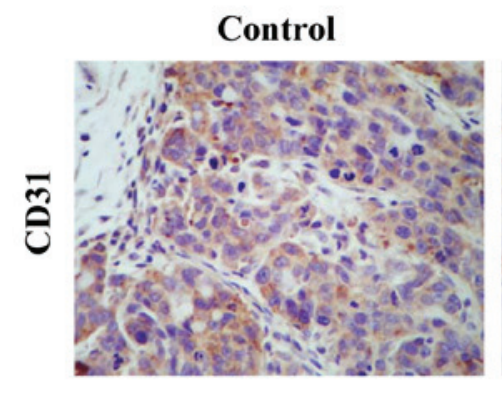

OA
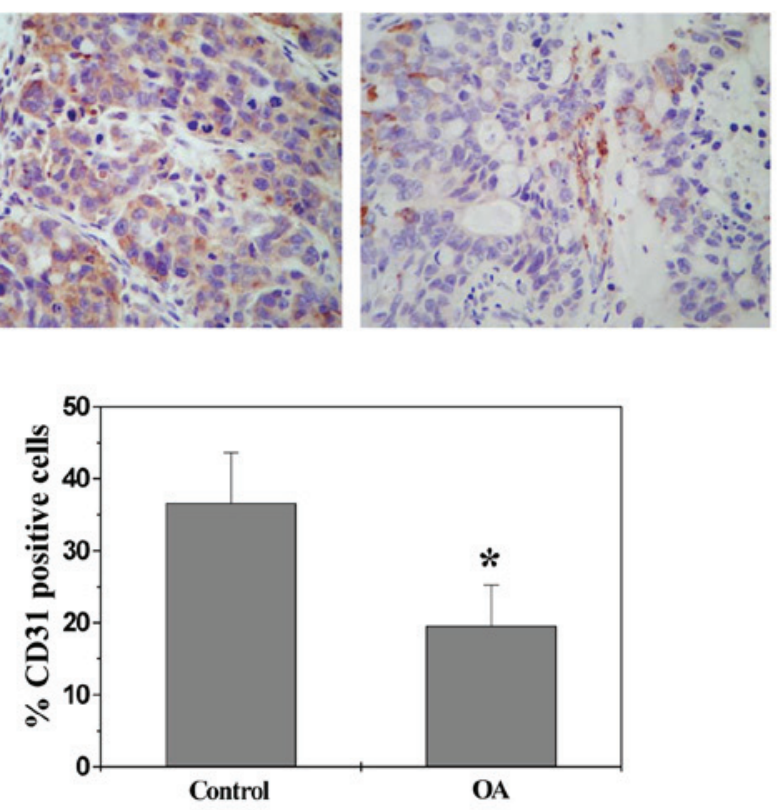

B
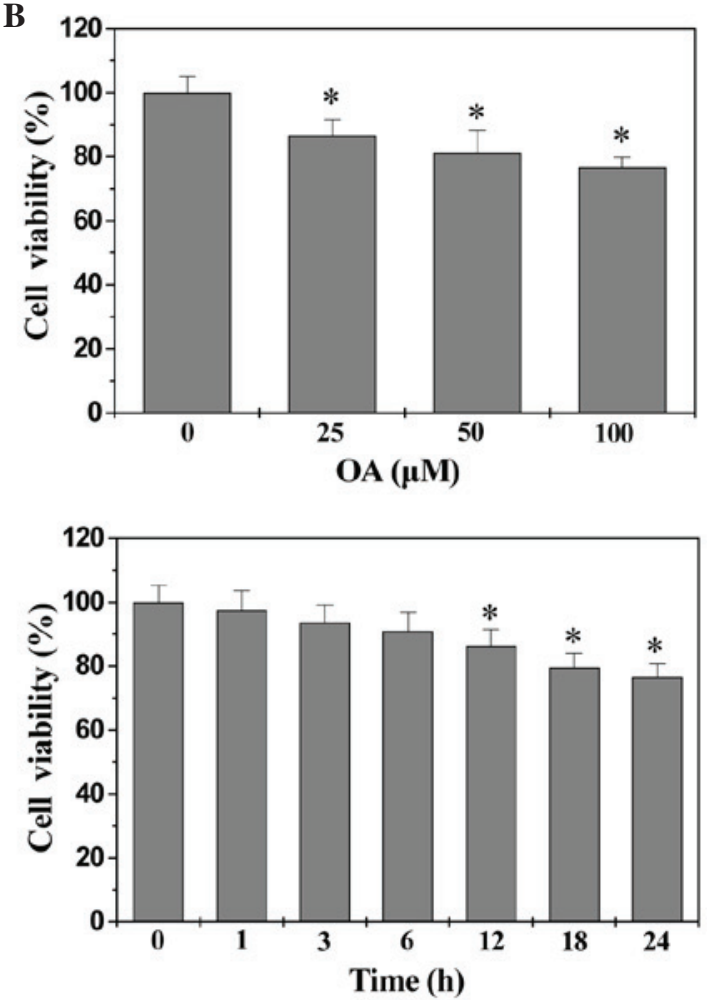

C
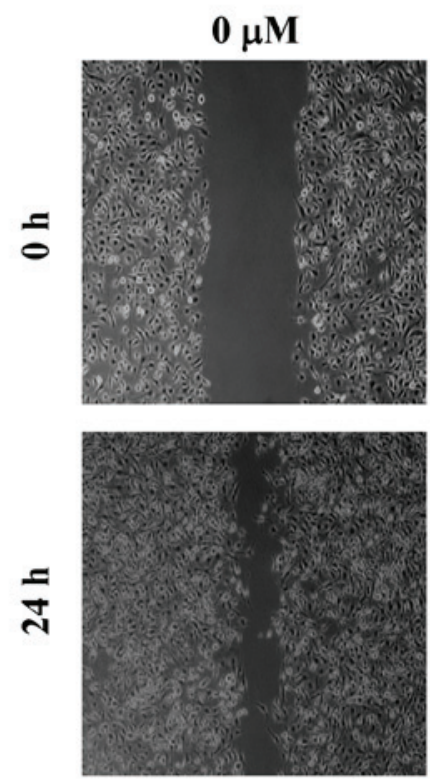

D
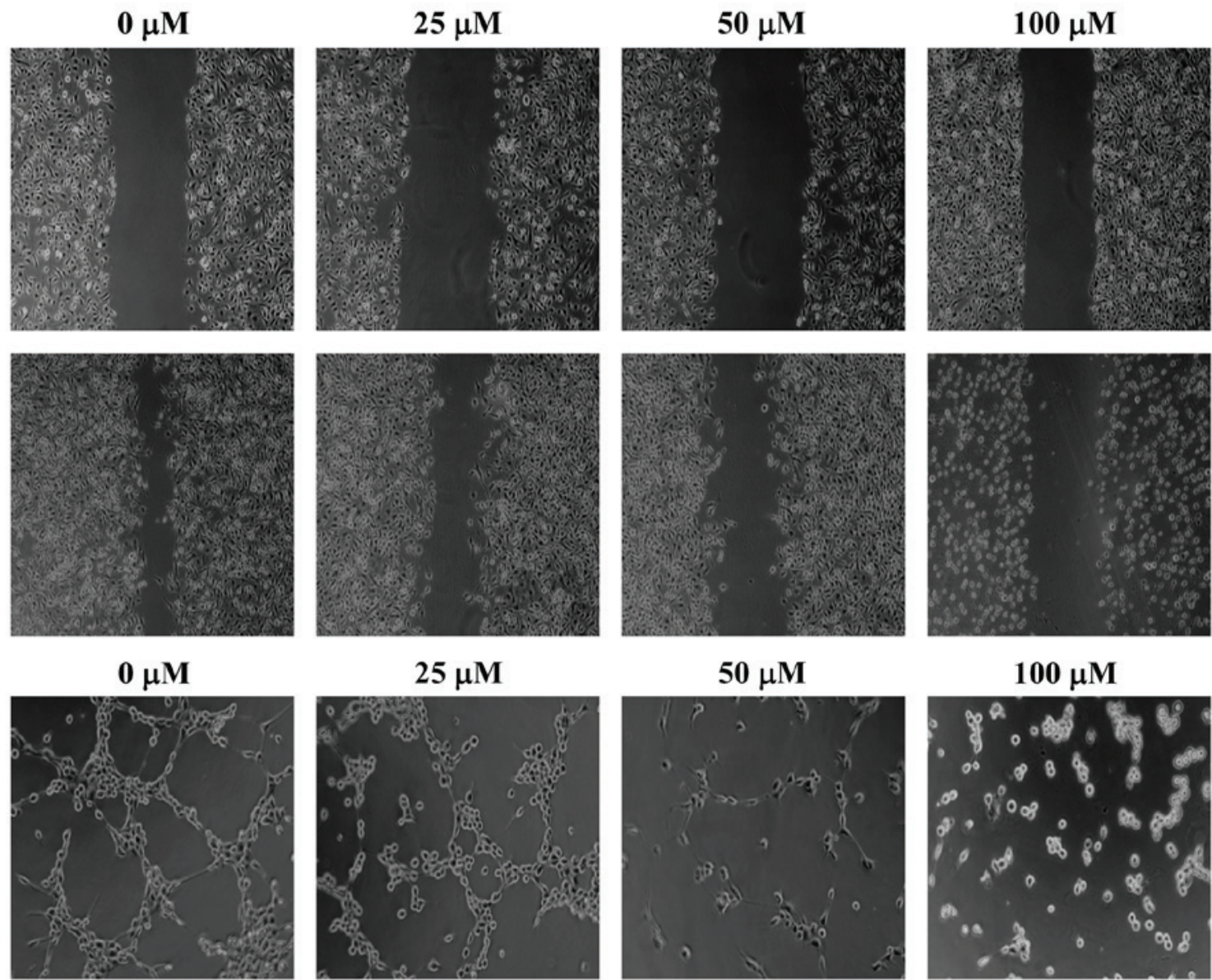

Figure 2. OA inhibited tumor angiogenesis. (A) IHS for CD31 in tumor tissues. Quantification of IHS was represented as percentage of positively-stained cells. Data are presented as the mean \pm standard deviation from eight individual mice in each group. Magnification, x400. (B) Cell viability of HUVECs was determined by the MTT assay. The data were normalized to the viability of control cells (100\%, treated with $0.1 \%$ dimethyl sulfoxide). Data are presented as the mean \pm standard error from three independent experiments. (C) Migration pattern and (D) tube formation of HUVECs was observed using phase-contrast microscopy. Images were representative of three independent experiments. Magnification, x100. * P $<0.01$ vs. the control group. OA, oleanolic acid; IHS, immunohistochemical staining; CD, cluster of differentiation; HUVECs, human umbilical vein endothelial cells. 
A

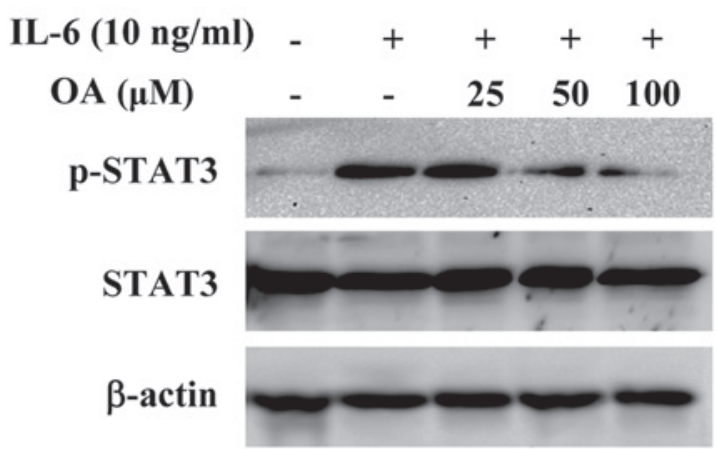

C

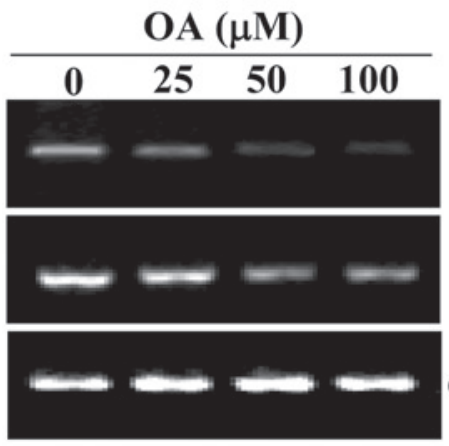

D

\section{SHH \\ Gli-1 \\ GAPDH}

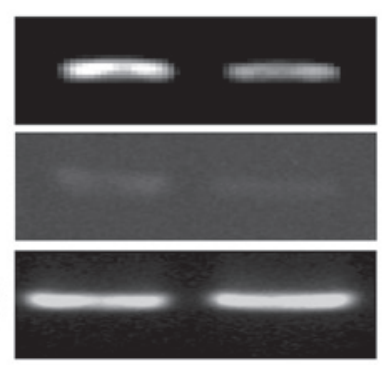

F
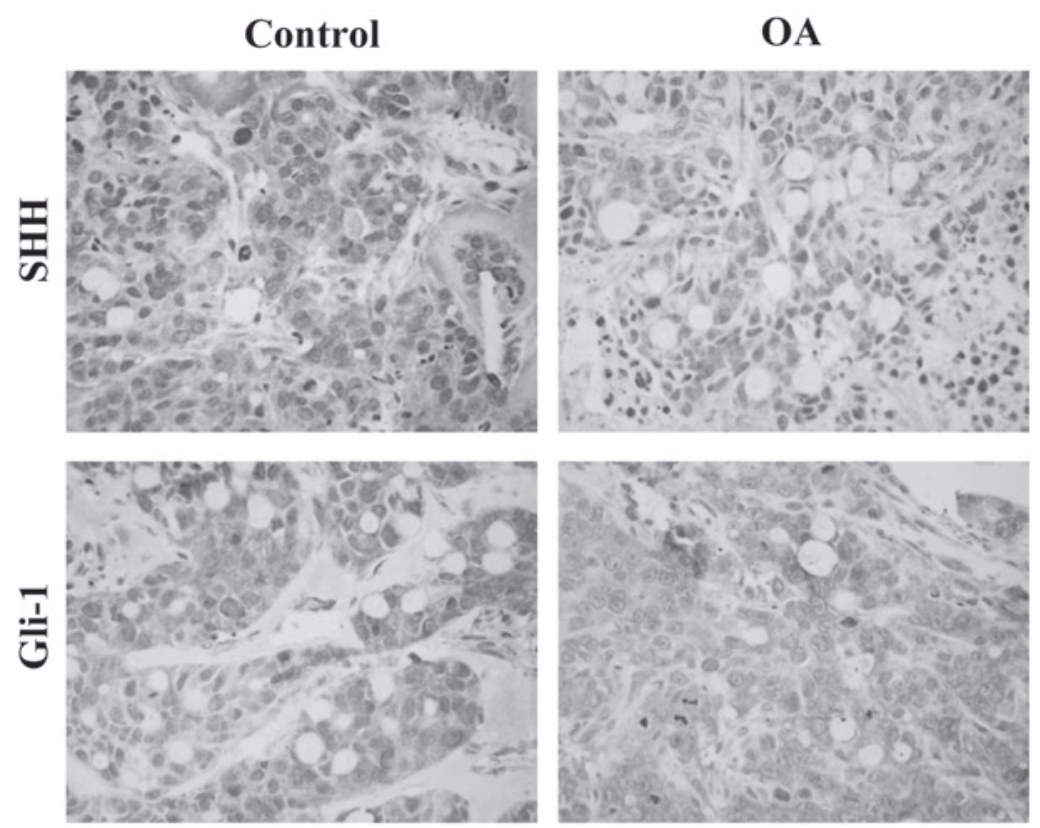

B

\section{p-STAT3}

STAT3

$\beta$-actin

\section{Control OA}
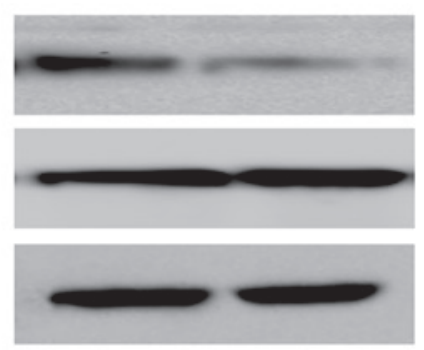

$\mathbf{E}$

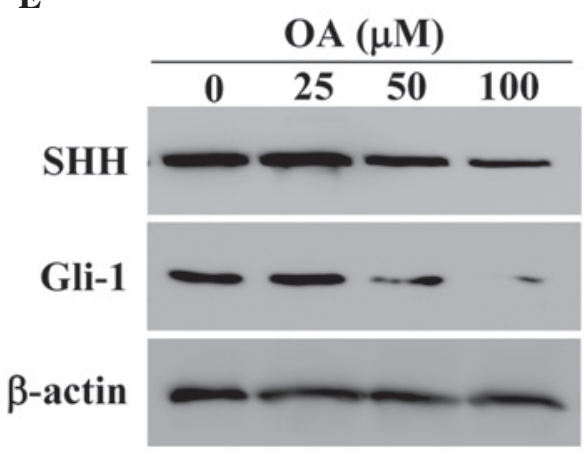

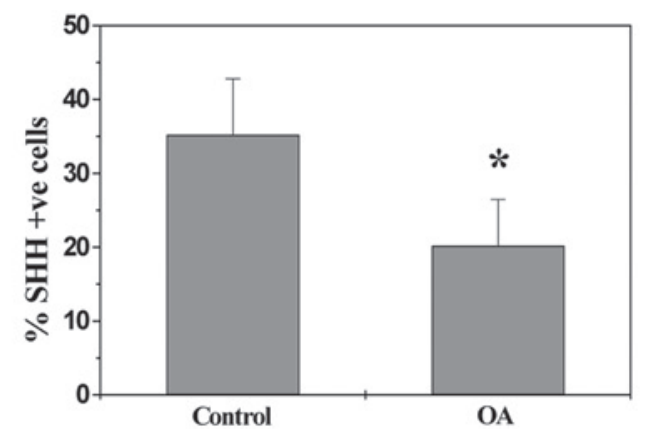

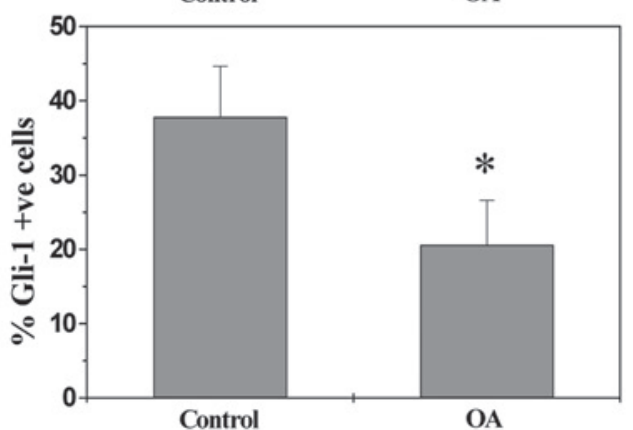

Figure 3. OA suppressed the activation of STAT3 and SHH pathways. STAT3 phosphorylation in (A) HT-29 cells and in (B) tumor tissues was determined by western blotting. The mRNA expression levels of SHH and Gli-1 in (C) HT-29 cells and in (D) tumor tissues were determined by RT-PCR. Protein expression levels of SHH and Gli-1 in (E) HT-29 cells was determined by western blotting. $\beta$-actin and GAPDH served as internal controls for western blotting and RT-PCR, respectively. Images were representative of three individual mice in each group or three independent cell-based experiments. Protein expression levels of SHH and Gli-1 in (F) tumor tissues was determined by immunohistochemistry. Images were representative and quantification data are presented as the mean \pm standard deviation from eight individual mice in each group. ${ }^{*} \mathrm{P}<0.01$ vs. the control group. Magnification, 400. IL-6, interleukin-6; OA, oleanolic acid; RT-PCR, reverse transcription-polymerase chain reaction; p, phosphorylated; STAT3, signal transducer and activator of transcription 3; SHH, sonic hedgehog; Gli-1, GLI-Kruppel family member GLI1.

OA suppresses STAT3 and SHH signalling pathways in vivo and in vitro. STAT3 activation was determined by western blotting using an antibody that targets STAT3 phosphorylation at $\mathrm{Tyr}^{705}$. As indicated by Fig. 3A and B, OA reduced
IL-6-induced phosphorylation of STAT3 in HT-29 cells and in tumors of CRC xenograft mice. The expression levels of non-phosphorylated STAT3 remained unchanged. The activation of SHH pathway was investigated by examining 
A

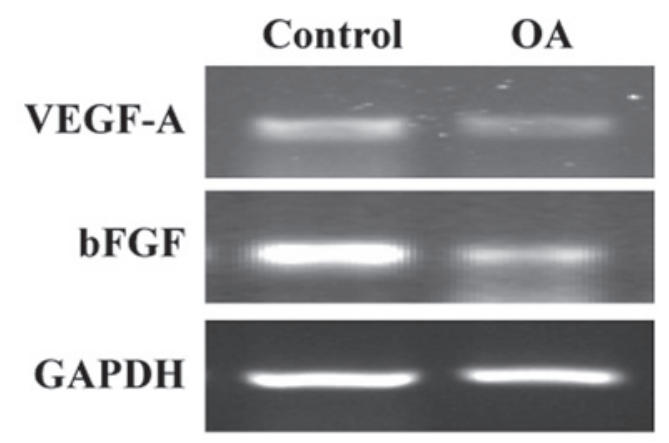

B

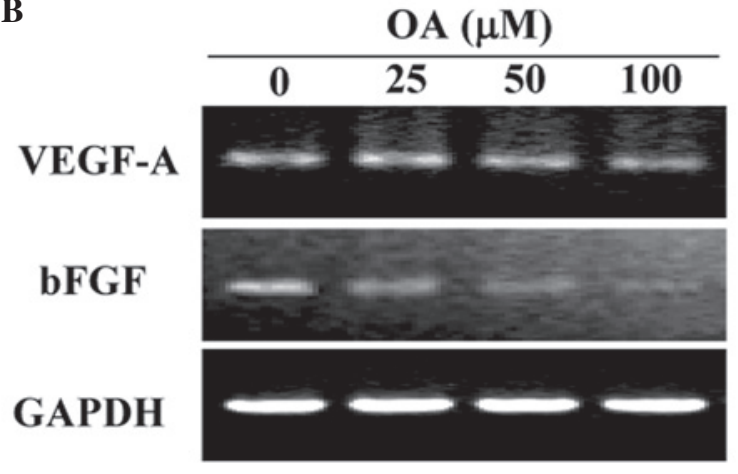

Figure 4. OA inhibited mRNA expression of VEGF-A and bFGF. The mRNA levels of VEGF-A and bFGF in (A) tumor tissues or in (B) HT-29 cells were determined by reverse transcription-polymerase chain reaction. GAPDH served as the internal control. Images captured were representatives of three individual mice in each group or three independent cell-based experiments. OA, oleanolic acid; VEGF-A, vascular endothelial growth factor A; bFGF, basic fibroblast growth factor.

the expression levels of the key mediators in CRC xenograft tumors and HT-29 cells. As indicated by Fig. 3C-F, OA treatment significantly reduced expression of $\mathrm{SHH}$ and Gli-1 in vitro and in vivo compared with the control $(\mathrm{P}<0.05)$, at the transcriptional and translational level.

$O A$ inhibits the expression of $V E G F-A$ and $b F G F$. The mRNA expression levels of VEGF-A and bFGF were determined by RT-PCR analysis. As indicated by Fig. 4, OA treatment decreased VEGF-A and bFGF mRNA expression levels in CRC xenograft tumor tissues and in HT-29 cells.

\section{Discussion}

Colorectal cancer (CRC) is one of the most common human malignant cancers. There are over 1 million newly diagnosed cases of CRC worldwide per year and >500,000 cancer-associated mortalities $(32,33)$. Chemotherapy is the predominant non-surgical therapeutic strategy for patients with advanced CRC. The majority of patients undergoing chemotherapy experience severe and debilitating side effects, which may be lethal in certain cases and may considerably outweigh the benefits for the patient $(34,35)$. In addition, long-term administration of the current chemotherapeutic agents often results in drug resistance (36). Thus, natural products may be an alternative as they produce fewer side effects and have been used extensively as viable alternative remedies for a variety of cancers (21-23). Various traditional Chinese medicinal herbs, including Hedyotic diffusa, Spica prunellae and Scutellaria barbata, have been used in clinical cancer treatment in China. OA is a bioactive compound present in these herbs and has been indicated to exert anti-cancer effects. However, the underlying mechanisms for these anti-tumor effects remain to be elucidated.

The present study determined that OA may significantly reduce intratumoral MVD in tumor tissue compared with the control $(\mathrm{P}<0.01)$, possibly via its inhibitory effect on multiple critical processes of angiogenesis, including proliferation, migration and capillary tube formation of endothelial cells. Therefore, OA may be an effective anti-angiogenic treatment in vivo and in vitro. Induction of angiogenesis is mediated by a variety of molecules released by tumor cells. VEGF-A and bFGF are considered to be strong stimulators of angiogenesis.
Overexpression of VEGF-A and bFGF has frequently been observed in various types of human cancer, which is associated with tumor progression (16-20). Upon binding to their specific receptors, VEGF-A and bFGF exert their biological function and trigger tyrosine kinase signaling cascades, resulting in endothelial cell proliferation and migration, and eventually capillary tube formation. Using RT-PCR analysis, the present study determined that OA treatment reduced mRNA expression levels of VEGF-A and bFGF in vivo in the CRC xenograft tumor tissues and in vitro in HT-29 cells.

The expression of VEGF-A and bFGF is regulated by multiple signal transduction cascades, including STAT3 and SHH pathways. STAT3 is a transcription factor, which is essential for numerous cellular processes. Constitutive activation of STAT3 is often associated with the development of numerous types of cancer and frequently indicates a poor prognosis (6-10). SHH is an extensively investigated member of the Hedgehog family and its aberrant activation has been associated with numerous types of human cancer, including CRC (11-15). OA treatment inhibited STAT3 phosphorylation and the expression of various key mediators of SHH signaling in CRC tumors and in HT-29 cells, indicating its suppressive effects on the activation of the STAT3 and SHH signaling pathways.

In conclusion, the present study demonstrated that inhibition of tumor angiogenesis via suppression of multiple signaling pathways may be one of the mechanisms by which OA exerts its anti-cancer function.

\section{Acknowledgements}

The current study was sponsored by the Research Fund for the Doctoral Program of Higher Education of China (grant no. 20133519110003) and the Developmental Fund of Chen Keji Integrative Medicine (grant nos. CKJ2014013 and CKJ2015007).

\section{References}

1. Folkman J: Angiogenesis. Annu Rev Med 57: 1-18, 2006.

2. Cook KM and Figg WD: Angiogenesis inhibitors: Current strategies and future prospects. CA Cancer J Clin 60: 222-243, 2010 . 
3. Mantovani A, Allavena P, Sica A and Balkwill F: Cancer related inflammation. Nature 454: 436-444, 2008.

4. Whiteside TL: The tumor microenvironment and its role in promoting tumor growth. Oncogene 27: 5904-5912, 2008

5. Kerbel RS: Tumor angiogenesis. N Engl J Med 358: 2039-2049, 2008.

6. Aggarwal BB, Kunnumakkara AB, Harikumar KB, Gupta SR, Tharakan ST, Koca C, Dey S and Sung B: Signal transducer and activator of transcription-3, inflammation and cancer: How intimate is the relationship? Ann NY Acad Sci 1171: 59-76, 2009.

7. Bromberg J and Wang TC: Inflammation and cancer: IL-6 and STAT3 complete the link. Cancer Cell 15: 79-80, 2009.

8. Kusaba T, Nakayama T, Yamazumi K, Yakata Y, Yoshizaki A, Inoue K, Nagayasu T and Sekine I: Activation of STAT3 is a marker of poor prognosis in human colorectal cancer. Oncol Rep 15: 1445-1451, 2006.

9. Lin Q, Lai R, Chirieac LR, Li C, Thomazy VA, Grammatikakis I, Rassidakis GZ,Zhang W, Fujio Y, Kunisada K, et al: Constitutive activation of JAK3/STAT3 in colon carcinoma tumors and cell lines: Inhibition of JAK3/STAT3 signaling induces apoptosis and cell cycle arrest of colon carcinoma cells. Am J Pathol 167: 969-980, 2005

10. Xiong H, Zhang ZG, Tian XQ, Sun DF, Liang QC, Zhang YJ, $\mathrm{Lu}$ R, Chen YX and Fang JY: Inhibition of JAK1, 2/STAT3 signaling induces apoptosis, cell cycle arrest and reduces tumor cell invasion in colorectal cancer cells. Neoplasia 10: 287-297, 2008.

11. Ingham PW, Nakano Y and Seger C: Mechanisms and functions of Hedgehog signalling across the metazoa. Nat Rev Genet 12 393-406, 2011

12. Theunissen JW and de Sauvage FJ: Paracrine Hedgehog signaling in cancer. Cancer Res 69: 6007-6010, 2009.

13. Yoshikawa K, Shimada M, Miyamoto H, Higashijima J, Miyatani T, Nishioka M, Kurita N, Iwata T and Uehara H: Sonic hedgehog relates to colorectal carcinogenesis. J Gastroenterol 44 1113-1117, 2009.

14. Varnat F, Duquet A, Malerba M, Zbinden M, Mas C, Gervaz P and Ruiz i Altaba A: Human colon cancer epithelial cells harbour active HEDGEHOG-GLI signalling that is essential for tumour growth, recurrence, metastasis and stem cell survival and expansion. EMBO Mol Med 1: 338-351, 2009.

15. Mazumdar T, DeVecchio J, Shi T, Jones J, Agyeman A and Houghton JA: Hedgehog signaling drives cellular survival in human colon carcinoma cells. Cancer Res 71: 1092-1102, 2011.

16. Kaya M, Wada T, Akatsuka T, Kawaguchi S, Nagoya S, Shindoh M, Higashino F, Mezawa F, Okada F and Ishii S: Vascular endothelial growth factor expression in untreated osteosarcoma is predictive of pulmonary metastasis and poor prognosis. Clin Cancer Res 6: 572-577, 2000.

17. Maeda K, Chung YS, Ogawa Y, Takatsuka S, Kang SM, Ogawa M, Sawada T and Sowa M: Prognostic value of vascular endothelial growth factor expression in gastric carcinoma. Cancer 77: 858-863, 1996.

18. Ferrara N, Gerber HP and LeCouter J: The biology of VEGF and its receptors. Nat Med 9: 669-676, 2003.

19. Ishigami SI, Arii S, Furutani M, Niwano M, Harada T, Mizumoto M, Mori A, Onodera $\mathrm{H}$ and Imamura M: Predictive value of vascular endothelial growth factor (VEGF) in metastasis and prognosis of human colorectal cancer. Br J Cancer 78 $1379-1384,1998$
20. Gille H, Kowalski J, Li B, LeCouter J, Moffat B, Zioncheck TF, Pelletier $\mathrm{N}$ and Ferrara N: Analysis of biological effects and signaling properties of Flt-1 (VEGFR-1) and KDR (VEGFR-2). A reassessment using novel receptor-specific vascular endothelial grow th factor mutants. J Biol Chem 276: 3222-3230, 2001.

21. Gordaliza M: Natural products as leads to anticancer drugs. Clin Transl Oncol 9: 767-776, 2007.

22. Newman DJ, Cragg GM and Snader KM: The influence of natural products upon drug discovery. Nat Prod Rep 17: 215-234, 2000.

23. Yang G, Li X, Li X, Wang L, Li J, Song X, Chen J, Guo Y, Sun X, Wang S, et al: Traditional chinese medicine in cancer care: A review of case series published in the Chinese literature. Evid Based Complement Alternat Med 2012: 751046, 2012.

24. Wei L, Lin J, Wu G, Xu W, Li H, Hong Z and Peng J: Scutellaria barbata D. Don induces G1/S arrest via modulation of p53 and Akt pathways in human colon carcinoma cells. Oncol Rep 29: 1623-1628, 2013.

25. Lin J, Wei L, Shen A, Cai Q, Xu W, Li H, Zhan Y, Hong Z and Peng J: Hedyotis diffusa Willd extract suppresses Sonic hedgehog signaling leading to the inhibition of colorectal cancer angiogenesis. Int J Oncol 42: 651-656, 2013.

26. Cai Q, Lin J, Wei L, Zhang L, Wang L, Zhan Y, Zeng J, Xu W, Shen A, Hong Z and Peng J: Hedyotis diffusa Willd inhibits colorectal cancer growth in vivo via inhibition of STAT3 signaling pathway. Int J Mol Sci 13: 6117-6128, 2012.

27. Peng J, Chen Y, Lin J, Zhuang Q, Xu W, Hong Z and Sferra TJ: Patrinia scabiosaefolia extract suppresses proliferation and promotes apoptosis by inhibiting the STAT3 pathway in human multiple myeloma cells. Mol Med Rep 4: 313-318, 2011.

28. Wang X, Bai H, Zhang X, Liu J, Cao P, Liao N, Zhang W, Wang Z and Hai C: Inhibitory effect of oleanolic acid on hepatocellular carcinoma via ERK-p53-mediated cell cycle arrest and mitochondrial-dependent apoptosis. Carcinogenesis 34: 1323-1330, 2013.

29. Lúcio KA, Rocha Gda G, Monção-Ribeiro LC, Fernandes J, Takiya CM and Gattass CR: Oleanolic acid initiates apoptosis in non-small cell lung cancer cell lines and reduces metastasis of a B16F10 melanoma model in vivo. PLoS One 6: e28596, 2011.

30. Wei J, Liu M, Liu H, Wang H, Wang F, Zhang Y, Han L and Lin X: Oleanolic acid arrests cell cycle and induces apoptosis via ROS-mediated mitochondrial depolarization and lysosomal membrane permeabilization in human pancreatic cancer cells. J Appl Toxicol 33: 756-765, 2013.

31. Institute of Laboratory Animal Resources (US). Committee on Care, Use of Laboratory Animals, and National Institutes of Health (US). Division of Research Resources: Guide for the care and use of laboratory animals. 8th edition. National Academies Press, Washington, DC, 2011

32. Siegel R, Desantis C and Jemal A: Colorectal cancer statistics, 2014. CA Cancer J Clin 64: 104-117, 2014.

33. Markowitz SD and Bertagnolli MM: Molecular origins of cancer: Molecular basis of colorectal cancer. N Engl J Med 361: 2449-2460, 2009.

34. Hanahan D and Weinberg RA: The hallmarks of cancer. Cell 100: 57-70, 2000.

35. Lippman SM: The dilemma and promise of cancer chemoprevention. Nat Clin Pract Oncol 3: 523, 2006.

36. Longley DB, Allen WL and Johnston PG: Drug resistance, predictive markers and pharmacogenomics in colorectal cancer. Biochim Biophys Acta 1766: 184-196, 2006. 